\title{
La integración de modalidades alternativas de capacitación a la presencialidad para el sector administrativo en la Universidad Nacional de Costa Rica
}

\section{The integration of alternative training modalities for the administrative sector at the National University of Costa Rica}

\author{
M.Sc. Marly Yisette Alfaro Salas ${ }^{1}$ \\ Universidad Nacional \\ M.Sc. Luis Diego Salas Ocampo² \\ Universidad Nacional
}

\section{Resumen}

El mundo de trabajo actual, las actividades programadas y no programadas, la atención de plazos inmediatos, así como la incorporación de la tecnología está obligando a las instituciones públicas y privadas a renovar las modalidades en las que sus sectores trabajadores pueden estar capacitados y actualizados de forma eficaz. Para ello, la incorporación de las metodologías novedosas, que permitan la transmisión del conocimiento de forma más accesible, es una necesidad imperante para cumplir este objetivo. En este campo, las

1 Administradora de Recursos Humanos, Gerencia y Negocios. Costarricense, Jefe Área de Formación y Actualización del Programa Desarrollo de Recursos Humanos, Académica, Escuela de Informática, Máster en Negocios, con estudios en Entornos Virtuales de Aprendizaje, estudiante del Doctorado en Ciencias de la Administración (malfaros@una.cr)

2 Sociólogo y administrador de empresas costarricense. Académico e investigador de la Universidad Nacional Autónoma de Costa Rica de la Escuela de Relaciones Internacionales. Máster en Entornos Virtuales de Aprendizaje y Administración de Negocios. Coordinador Proyecto Fortalecimiento de las Capacidades de Investigación mediante el uso de software cuantitativos, cualitativos y simuladores de negocios, estudiante del Doctorado en Ciencias de la Administración (Isala@una.cr) 
capacitaciones en las que predomina la presencialidad están siendo relegadas por otras metodologías que incorporan los entornos virtuales, se convierten en un referente de liderazgo tecnológico en la gestión de la capacitación. Se evidencia, en este trabajo, la necesidad de que la universidad genere metas que orienten el desarrollo de un enfoque de e-learning a nivel de capacitación y realice los cambios para llegar a ese nivel. Al menos, debe ubicar el tema dentro de la estrategia institucional, cambiar los mecanismos de formación de docentes y promover planes de internacionalización con los programas que inicien este tipo de cambios. Es clave que la estructura administrativa de la universidad tenga su propia estrategia de capacitación que apunte a estos insumos que se plantean.

Palabras claves: Estructura administrativa; virtualidad; bimodalidad; actividades formativas; e-learning; capacitación.

\section{Abstract}

The current working world, the activities scheduled and unscheduled, care of immediate deadlines, and the incorporation of technology is forcing public and private institutions to renew the arrangements under which workers can be trained and updated on a effective. To this end, the incorporation of new methodologies that allow the transmission of knowledge in a more accessible, is still a pressing need to meet this objective. In this field it is that trainings in which predominantly presentiality are being relegated by other methodologies that incorporate virtual environments, become a benchmark for technological leadership in management training.

That is why the need for educating and concludes that it is necessary that the University define what possibly could achieve working toward developing an e-learning approach and the changes you must do to reach that level is evident. At least, you must place the issue within the corporate strategy, change mechanisms teacher training and promote internationalization plans with programs that initiate such changes. It is key that the administrative structure of the University has its own training strategy that targets these inputs arise.

Key Words: Administrative structure; virtuality; bimodalidad; formative activities; e-learning; training 


\section{Introducción}

La transmisión del conocimiento ha sido todo un reto para el sector educativo formativo, el cual, con el cambio del tiempo, la tecnología, las modalidades, las estructuras organizativas, entre otros factores, comprueba la necesidad de incorporar metodologías y herramientas más efectivas para poder cumplir con el reto de formar y desarrollar el talento humano.

En el campo del uso de la virtualidad o educación a distancia, en Costa Rica el proceso ha iniciado primeramente con la educación a distancia en 1970 y ya en 1990, se presenta todo un movimiento de institucionalización de las tecnologías de información y comunicación (TIC) dentro de los distintos ámbitos institucionales de la educación universitaria.

Los datos refuerzan la idea de una oferta cada vez mayor de educación en este modelo virtual tanto a nivel privado como público, por ejemplo, la Contraloría General de la República, en su plan para el año 2012, integra 113 actividades de desarrollo, con métodos de aprendizaje de capacitación presencial y en línea. Las proyecciones de esta iniciativa establecen que, con este número de actividades, serán capacitados 4305 funcionarios con un total de 2768 horas (Centro de Cooperación Regional para la Educación de los Adultos en América Latina y el Caribe, 2012). Es claro que también en este contexto se presenta una diversificación de la naturaleza de las actividades con las cuales se capacita, promoviendo la utilización de foros, webmeetings, talleres, videoconferencias, asesorías en el sitio y algunos otros.

En este contexto, son varios los cuestionamientos que pueden y deben hacerse en relación con las metas que debemos asumir como instituciones públicas. Concretamente, este trabajo se centra desde la experiencia de la Universidad Nacional Autónoma de Costa Rica (UNA), en cuanto a: ¿Cómo ha capitalizado su experiencia de cara a un fortalecimiento de todo su aparato institucional en su conjunto tanto a nivel de recurso de soporte administrativo, como académico? ¿Es posible pensar que se pueda integrar la virtualidad en las actividades formativas? 


\section{Virtualidad dentro de las estructuras administrativas de las universidades}

Es ya sumamente conocida la premisa antigua de Meyer según la cual las condiciones de liderazgo afectan de manera importante las variables definitorias de la estructura organizacional. Este autor menciona que, donde los liderazgos en el pasado han cambiado y donde se suelen tener relaciones de dependencia y comunicación cercana entre personal y los niveles directivos más altos de la organización, la estructura tenderá a la inestabilidad.

Dicho de otra manera, si aplicáramos las ideas de este profesor de la Universidad de California a las premisas que se tienen de las universidades en el campo de la economía del conocimiento para el caso nacional, podría llegarse a la conclusión que señala que si no existe la sensibilización dirigida hacia los sectores administrativos sobre el papel de la tecnología y la innovación para el mejoramiento de los procesos y el aumento de la competitividad, el rol de la universidad como enlace con la economía global de conocimientos, como espacio de acercamiento de sectores sociales y como generadora de líderes, podría verse afectado negativamente.

Adicionalmente, dentro de los planteamientos de las universidades costarricenses, en particular la UNA, el campo del aprendizaje no está solamentelimitadoalsectoracadémico, yaquedentrodesusestrategias de acción se incluyen a empresas privadas, organizaciones sociales, educación a adultos, educación continua dirigida tanto a personal académico como administrativo, entre otros. Además de la necesidad que han venido demandando las autoridades universitarias en torno a establecer prioridades de formación y actualización, debidamente fundamentas, que vengan a responder a necesidades o diagnósticos técnicamente para poder capacitar al sector administrativo.

\section{Metodología}

Se parte del principio de que para determinar el nivel de integración que una universidad posee es necesario establecer 
tanto el nivel de uso como la intensidad que tiene la educación virtual y el e-learning dentro de la lógica de estructuración de la oferta educativa en sus diferentes modalidades y en función de actores claves, tales como los sectores académicos, así como los administrativos, por las razones anteriormente expresadas.

Ello llevó a que esta investigación partiera de la recuperación de datos de fuentes primarias de la propia Universidad Nacional que permitieran establecer tanto cómo y con qué intensidad se piensa en educación virtual, principalmente en lo relacionado con actividades formativas.

Algunas de las fuentes con las que se trabajaron fueron:

- Listado de personal administrativo proporcionado por el Programa Desarrollo de Recursos Humanos (PDRH)

- Cursos impartidos desde el Área de Formación y Actualización del Talento Humano (PDRH)

- Diccionario de competencias

- Manual de perfiles de competencias

Los datos han sido recopilados en el primer ciclo del 2015, en particular en el mes de junio. Este es un estudio, por lo tanto, transversal, donde los resultados explican el comportamiento de este fenómeno en este punto en el tiempo. Adicionalmente, el tratamiento de los datos es de corte descriptivo, sobre todo, por la dificultad de encontrar una única base de datos que logre condensar las variables de interés del estudio.

Las principales variables trabajadas son:

- Presencia de educación con entornos virtuales en las capacitaciones dirigidas al sector administrativo y cargos de gestión académico-administrativa

- Competencias institucionales deseables en el sector administrativo de la Universidad Nacional 
- Número de personal administrativo según planilla al mes de mayo de 2015

- Relación estrato profesional en cuanto al total de personal administrativo

- Actividades de capacitación promovidas desde el Área de Formación y Actualización del Talento Humano al mes de octubre de 2015

Estas variables en su conjunto permiten hacer un cuadro que lleva a visualizar tanto el nivel de importancia de la educación virtual en la Universidad y, en este tanto, a poder dar una idea preliminar sobre las posibilidades de esta casa de estudios de desarrollar, a nivel de país, un rol trascendente en materia de formación mediante entornos virtuales.

\section{Análisis de resultados}

De acuerdo con el concepto de economía de conocimiento, las universidades tienen al menos tres roles fundamentales: como espacio de acercamiento de sectores sociales que conciben el desarrollo; como punto de enlace con otras instancias productoras de conocimientos en el escenario global, y como organización formadora de líderes.

El punto clave del análisis se centra en la educación virtual como primer eslabón de un proceso de entornos innovadores de capacitación. Es por ello que realizar un análisis de la implementación de los programas de capacitación institucional, mediante la virtualidad y el uso de la tecnología como herramientas de aprendizaje en la Universidad Nacional, constituye el primer ejercicio básico de abordaje del tema.

Con la implementación a partir de 01 de mayo del año 2007 del Modelo de gestión por competencias, se ha buscado la integración del saber hacer en la gestión administrativa, desde los cargos operativos hasta los de Dirección. 
Junto con el modelo, se aprueba a su vez el Diccionario de competencias, el cual debe actualizarse constantemente, debido a que describe cada una de las capacidades, habilidades y pensamientos requeridos en el conjunto de perfiles de cargos propuestos en la Institución. Para el año 2011 se aprueba esta herramienta ${ }^{3}$ que permite, entre otras cosas, la definición de los planes de desarrollo, al ser las evidencias factores por considerar en el momento de establecer la temática por trabajar y los segmentos de funcionarias y funcionarios universitarios por desarrollar.

Asimismo, se generan los insumos para crear indicadores de evaluación genéricos, tanto en la determinación como en el cierre de brechas, relacionados con los ejes temáticos de formación y desarrollo. Precisamente la última edición del Diccionario de competencias, agrupa los cargos administrativos en un total de diez competencias, las cuales se indican seguidamente:

\section{Tabla 1}

Competencias institucionales para el sector administrativo de la UNA al 2015

\section{Institucionales}

Refieren a las competencias generales, las cuales deben estar presentes en todos los colaboradores del sector administrativo y están relacionados con las características esenciales o valores que deben

1. Actitud de servicio

2. Trabajo para el equipo distinguir al personal universitario.

3. Actitud de mejora

\section{Específicas}

Describen características personales que predicen

4. Organización

5. Planificación un desempeño satisfactorio en la ejecución de

6. Liderazgo funciones según rol asignado.

7. Comunicación

8. Disposición para el cambio

\section{Técnicas}

Representan los conocimientos, habilidades y destrezas inherentes a un desempeño exitoso en el cargo, así como evidencias requeridas para el desempeño de las funciones y actividades descritas en cada uno de los

9. Análisis

10. Dominio y aplicación técnica macroprocesos, estratos, niveles y perfiles de cargo.

Nota: Elaboración propia, según cuadro del Diccionario (2012).

3 Aprobada la primera edición, por la Vicerrectoría de Desarrollo, mediante informe PDRH-OTCVC-I-004-2011, del 23 de noviembre del 2011 y la segunda edición PDRH-OTCVC-IA-2-2012 del 21 de noviembre de 2012. 
A partir de la aprobación de este Diccionario y como producto de la evaluación del Modelo de Gestión por Competencias que se realiza durante el año 2012, se crea una Comisión de Desarrollo, la cual tiene como fin el revisar y actualizar los contenidos y modalidades para la capacitación del personal administrativo. Sin embargo, a la fecha, solamente se ha realizado una capacitación en forma virtual a este sector, para las demás capacitaciones se mantiene el modelo tradicional presencial para recibir estas formaciones complementarias.

Es importanteconsiderar que las competencias a niveladministrativo están estructuradas cada una por tres grados, lo que significa que, eventualmente, se requerirían al menos 30 capacitaciones para el sector administrativo que está involucrado. De estas, 9 (de las 3 competencias institucionales) son inherentes a todos los cargos de la institución, a saber, trabajo para el equipo, actitud de mejora y actitud de servicio. Por otra parte, se siguen realizando capacitaciones presenciales con fines participativos, no formativos, es decir, de aprovechamiento. En este sentido, el uso del entorno virtual (e-learning) sería una modalidad que vendría a responder a estas necesidades, abarcar más personas y poder establecer mecanismos efectivos de control y desarrollo de la competencia.

En este sentido es valioso considerar la clasificación general de los cargos del sector administrativo, en relación con los denominados estratos operativos, técnicos, profesionales o de dirección, tal como se visualiza en la figura 1.

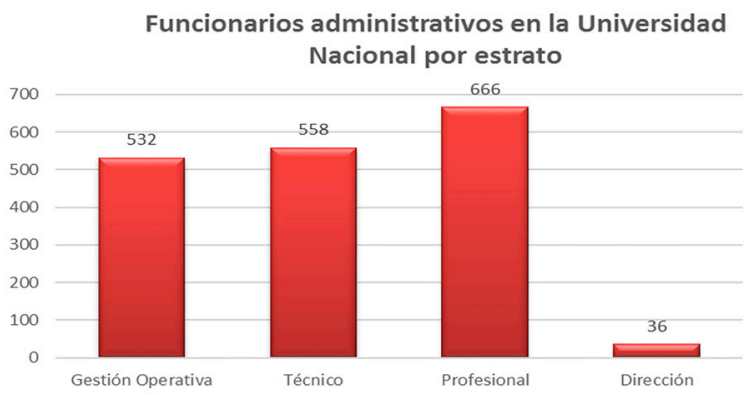

Figura 1. Cantidad de personal administrativo por estrato ocupacional en la Universidad Nacional de Costa Rica. Elaboración propia con base en datos de Sistemas de información NX, UNA, nombramientos ejecutados al mes de mayo 2015. 
Tal como se evidencia en la figura 1, la población administrativa con más representación es la que se agrupa en el sector profesional y la segunda es la técnica. Se consideran estos estratos como referencia por cuanto, por las funciones y actividades propias de estos cargos, en su mayoría tienen contacto con la tecnología y, por tanto, podrían ser considerados en modelos de capacitación en otras modalidades no presenciales.

En esto es importante considerar lo que porcentualmente significa el tiempo presencial de funcionario en procesos de capacitación, según poblaciones por estrato y totalidad de funcionarios, es decir, si se capacitara al $20 \%$ de la población administrativa en cargos profesionales y técnicos, en cursos de capacitación de 20 horas (sea por medio del Área de Formación y Actualización del Talento Humano, recursos de las unidades académicas o bien por la Junta de Becas), durante el año se estaría desligando de su trabajo a 245 personas aproximadamente, para un total de 4900 horas, solamente para la asistencia a las capacitaciones, esto sin considerar los tiempos adicionales para trabajos o tareas, repaso de materiales o incluso el proceso adicional de evaluación de la capacitación. Esto, de acuerdo con presupuesto laboral, equivaldría a $1.71 \%$ plaza tiempo completo anual; números que podrían parecer mínimos, pero si se considera el costo real de estas horas, su efecto es mucho mayor.

Para estos estratos, como muestra general, el tener otras modalidades para poder ser capacitados, sería enriquecedor no solo para la persona, sino para la institución, en relación con el costo-beneficio de la integración de la bimodalidad o la virtualidad en las actividades formativas.

Sin embargo, al analizar las actividades de formación promovidas durante el año 2015 por medio del Área de Formación y Actualización del Talento Humano, se encuentra la siguiente programación (tabla 2). 
Tabla 2

Actividades formativas promovidas desde el Área de Formación del Talento Humano (PDRH)

\begin{tabular}{|c|c|c|c|c|}
\hline $\begin{array}{l}\text { Nombre actividad } \\
\text { formativa }\end{array}$ & $\begin{array}{c}\mathrm{N}^{\circ} \\
\text { grupos }\end{array}$ & $\begin{array}{c}\mathrm{N}^{\circ} \\
\text { convocados }\end{array}$ & $\begin{array}{l}\text { Horas } \\
\text { por } \\
\text { grupo }\end{array}$ & $\begin{array}{l}\text { Horas } \\
\text { totales }\end{array}$ \\
\hline Redacción de informes & 2 & 25 & 40 & 80 \\
\hline $\begin{array}{l}\text { Redacción de informes } \\
\text { avanzado }\end{array}$ & 4 & 50 & 40 & 160 \\
\hline Trabajo para el equipo & 2 & 25 & 16 & 32 \\
\hline $\begin{array}{l}\text { Planificación en la } \\
\text { gestión del trabajo } \\
\text { Gestión Pública en }\end{array}$ & 4 & 100 & 16 & 64 \\
\hline Instancias Académicas & 2 & 35 & 68 & 130 \\
\hline $\begin{array}{l}\text { Mejora continua en el } \\
\text { desempeño }\end{array}$ & 2 & 20 & 16 & 32 \\
\hline Formación de formadores & 1 & 15 & 16 & 16 \\
\hline Organización del trabajo & 1 & 25 & 16 & 16 \\
\hline $\begin{array}{l}\text { Métodos y técnicas de } \\
\text { investigación }\end{array}$ & 1 & 30 & 16 & 16 \\
\hline $\begin{array}{l}\text { Excelencia en el servicio } \\
\text { al usuario } \\
\text { Actitud de mejora, grado }\end{array}$ & 1 & 25 & 16 & 16 \\
\hline 2 & 1 & 18 & 16 & 16 \\
\hline Organización, grado 2 & 1 & 18 & 16 & 16 \\
\hline $\begin{array}{l}\text { Trabajo para el equipo, } \\
\text { grado } 2 \\
\text { Talento Humano en la }\end{array}$ & 1 & 18 & 16 & 16 \\
\hline UNA & 1 & 30 & 16 & 16 \\
\hline $\begin{array}{l}\text { Calidad y Servicio al } \\
\text { usuario }\end{array}$ & 1 & 25 & 16 & 16 \\
\hline $\begin{array}{l}\text { Administración del } \\
\text { Tiempo }\end{array}$ & 2 & 70 & 16 & 32 \\
\hline $\begin{array}{l}\text { Coordinación técnica de } \\
\text { equipos de trabajo }\end{array}$ & 2 & 70 & 16 & 32 \\
\hline $\begin{array}{l}\text { Enfoque, Diseño, } \\
\text { Modelos de Investigación }\end{array}$ & 4 & 110 & 32 & 128 \\
\hline Organización de eventos & 1 & 30 & 16 & 16 \\
\hline Gestión de expedientes & 1 & 25 & 16 & 16 \\
\hline Tipos documentales & 1 & 25 & 60 & 60 \\
\hline Comunicación asertiva & 4 & 115 & 12 & 48 \\
\hline Total & 40 & 904 & 508 & 980 \\
\hline
\end{tabular}

Nota: Actividades formativas ejecutadas a octubre de 2015. Elaboración propia, según listados de convocatorias a cursos. 
Estas actividades están dirigidas principalmente al sector administrativo y académico en gestión administrativa; las 22 actividades abarcaron en convocatoria a 904 funcionarios. De los cursos, 16 estaban dirigidos a cargos profesionales de los diferentes macroprocesos y dos a académicos en gestión administrativa. Es decir, que la asistencia únicamente en modalidad presencial a estas capacitaciones implica que procesos clave de la institución puedan verse afectados; sin embargo, las mismas son necesarias para el desarrollo del personal.

No obstante la necesidad de implementar entornos virtuales, la implementación de estos no es evidente en las actividades de capacitación mediadas por el Área de Formación y Actualización del Talento Humano, lo que se muestra en la tabla 3.

\section{Tabla 3}

Actividades formativas e incorporación de los recursos virtuales en la UNA

\begin{tabular}{|l|c|c|c|c|c|c|c|}
\hline $\begin{array}{l}\text { Nombre actividad } \\
\text { formativa }\end{array}$ & $\begin{array}{c}\mathbf{N}^{\circ} \\
\text { grupos }\end{array}$ & $\begin{array}{c}\text { Apoyo } \\
\text { Tecnológico }\end{array}$ & $\begin{array}{c}\text { Sesiones } \\
\text { virtuales }\end{array}$ & Bimodal & $\begin{array}{c}\text { Horas del } \\
\text { curso }\end{array}$ & $\begin{array}{c}\text { Horas no } \\
\text { presenciales }\end{array}$ & $\begin{array}{c}\text { Relación } \\
\%\end{array}$ \\
\hline $\begin{array}{l}\text { Gestión Pública en } \\
\text { Instancias Académicas }\end{array}$ & 2 & SI & NO & NO & 136 & 0 & $0 \%$ \\
\hline $\begin{array}{l}\text { Calidad y servicio al } \\
\text { usuario }\end{array}$ & 1 & DI & SI & SI & 16 & 5 & $50 \%$ \\
\hline $\begin{array}{l}\text { Enfoque, Diseño, Modelo } \\
\text { de Investigación }\end{array}$ & 4 & SI & SI & NO & 128 & 32 & $25 \%$ \\
\hline \multicolumn{1}{c|}{ Total } & & & & & 280 & 40 & $14,3 \%$ \\
\hline
\end{tabular}

Nota: Actividades formativas ejecutadas a octubre de 2015. Elaboración propia, según programas de cursos.

De las 22 actividades formativas, que significaron 40 grupos de capacitación y 1862 horas de formación, solamente en tres se implementó el uso de entornos virtuales, para un total de 40 horas efectivas no presenciales, lo que significa que solamente el $2,15 \%$ de las horas fueron con modalidades diferentes a las presenciales. No obstante, llama la atención que, de los 22 cursos, dos de ellos implementaron la virtualidad y uno fue bimodal. Es de especial interés el curso de Gestión Pública en Instancias Académicas, 
que está dirigido a cargos de gestión: direcciones, subdirecciones y jefaturas que, aunque utilice el aula virtual, esta se convierta en repositorio de documentos, no así en una herramienta para que los cursos no sean predominante presenciales.

Finalmente, aunque no menos importante, según los informes de curso y las personas que obtienen certificado de aprovechamiento o participación, el hecho que las formaciones sean principalmente en la presencialidad, podría hacer que el grado de deserción sea mayor que si las capacitaciones integraran la bimodalidad o virtualidad; esto, por cuanto, en el único curso que se convocó como bimodal, las personas que asistieron a la primera sesión, finalizaron el curso satisfactoriamente.

\section{Conclusiones}

La institución ha dado pasos importantes en la integración de la virtualidad, en los procesos de enseñanza; muestra de ello es que se cuenta con un programa que está enfocado directamente a la virtualidad como medio de aprendizaje. El Modelo Pedagógico Universitario, busca entre otras cosas:

... diversos espacios de enseñanza y aprendizaje (aulas, laboratorios, gimnasios, aulas virtuales, bibliotecas, entre otros) confluyen personas con diversos estilos, con experiencias propias de sus contextos, con diferentes capacidades, lo que requiere variedad en los procesos de mediación pedagógica y la utilización de diversas estrategias... (p. 12)

Como reflejaron las tablas 3 y 4 , aunque el discurso de las autoridades promueva metodologías novedosas para la capacitación, casi el $98 \%$ de las horas recibidas son presenciales. Este comportamiento eleva el costo económico de los procesos de capacitación. Si se 
considera solo el costo - hora del funcionario o funcionaria que se desplaza de su trabajo al lugar de la capacitación y se suman otros costos asociados, por ejemplo, la alimentación en caso de que se facilite como refrigerio, es claro este comportamiento. Además, el desplazamiento para capacitarse tiene un costo de oportunidad en la unidad académica o administrativa, ya que tareas de seguimiento, control y gestión de los procesos que este ejecuta presencialmente en la instancia, no podrán ser realizados.

Esto evidencia, entonces, la necesidad de una mayor preparación de parte de del personal del Área de Formación y Actualización del Talento Humano en metodologías de enseñanza y entornos virtuales, lo que implica apoyo tanto de las autoridades, como del Programa de Evaluación Académica y Desarrollo Profesional para facilitar la incorporación de profesionales administrativos en cursos destinados a desarrollar las metodologías de educación y el uso del aula virtual.

En un segundo momento está la alfabetización tecnológica de las funcionarias y funcionarios administrativos, con el fin de que conozcan los recursos virtuales, el uso del aula virtual o bien otros recursos tales como las herramientas disponibles. Este aspecto requerirá la integración del Área de Formación y Actualización del Talento Humano como mediadora de este aprendizaje; sin embargo, debido a la capacidad instalada del área, esta tarea podrá llevarse a cabo con el apoyo de otras instancias universitarias con las capacidades requeridas.

En este campo, es importante que se pueda tener un registro interno de personas facilitadoras que puedan participar en estos procesos de formación, que tengan formación en pedagogía y entornos virtuales, de esta manera será más efectiva la transición de la presencialidad - bimodal, bimodal-virtual y presencial-virtual.

Finalmente, con el fin de poder implementar la virtualidad o bimodalidad, como primer paso es necesario que se facilite la capacitación institucional con unaagresiva promoción demetodologías no presenciales de capacitación. Por ello, se recomienda: 
1. Que la Vicerrectoría de Docencia, con base en el Modelo Pedagógico, puede jugar un rol fundamental en este cambio cultural, por medio de un modelo educativo integrado que acuerpe el Sistema de Desarrollo Profesional, tanto administrativo como académico.

2. Que, por medio del Programa de Desarrollo de Recursos Humanos, se pueda promover y continuar facilitando la formación de su personal profesional, en una primera fase del Área de Formación y Actualización del Talento Humano, en pedagogía y entornos virtuales.

3. Que el Área de Formación y Actualización del Talento Humano incorpore la bimodalidad o virtualidad como un requerimiento esencial en los procesos de contratación de capacitaciones para el sector administrativo. Esto por cuanto a la fecha se promueven como opcionales.

4. Finalmente, que las capacitaciones que el Programa UNA-Virtual realice se hagan en forma virtual, para que más personas usuarias puedan conocer las tecnologías de información disponibles y las puedan utilizar.

\section{Referencias}

Area, M. (2013). Formación virtual en e-learning, Potencialidades, limitaciones y destrezas. Recuperado de http://ordenadoresenelaula.blogspot. com/2013/01/la-formacion-virtual-o-elearning.html

Centro de Capacitación de la Contraloría General de la República. (2012). Plan de capacitación externa. San José, Costa Rica

Centro de Cooperación Regional para la Educación de los Adultos en América Latina y el Caribe. (2012). Generación y gestión del conocimiento. Recuperado de http://www.crefal.edu.mx/crefal2012/

De Benito, B. (2000). Posibilidades educativas de las "webtools". Mallorca: Universidad de las Islas Baleares.

Galbreath, J. (1997). The Internet: past, present, and future. Educational Technology, 39-44. Recuperado de: http://historia.ucr.ac.cr/repositorio/ bitstream/123456789/74/1/working $\% 20$ paper\%20nuevas $\% 20$ tic $\% 20$ en\%20america\%20central-\%20jmarin.pdf 
Maestría en Diseño de Entornos Virtuales de Aprendizaje. (s. f.). La maestría en Diseño de Entornos Virtuales de Aprendizaje http://campus.crefal.edu.mx/campusv/index.php?option=com content $\&$ view $=$ article $\&$ id $=83 \&$ Itemid $=2$

Marín, J. (2012). Balance y perspectivas de la educación a distancia en Centroamérica en el contexto de la bimodalidad. Recuperado de https:// www.academia.edu/1604999/Working_paper_sobre_las_TICs_en_ Centroam\%C3\%A9rica

Meyer, B. J. (1975). The organization of prose and its effects on memory (Vol. 1). Amsterdan, Holland, North-Holland Publishing.

Programa Desarrollo de Recursos Humanos Maestría en Educación a Distancia (2015). Listado de funcionarios administrativos. Recuperado de http:// www.uned.ac.cr/posgrado/index.php/maestrias/102-educacion-adistanciaSiete tendencias del E-learning (s. f.). Recuperado de http:// www.libro-elearning.com/tdt-mundo-educativo-tlearning.html

Universidad Nacional (s. f.). Listado de actividades formativas mediadas por el Área de Formación y Actualización del Talento Humano, al mes de octubre de 2015.

Universidad Nacional (s. f.). Oferta académica UNA. Recuperado de http:// www.una.ac.cr/docencia/index.php?option=com_content\&task=view\&i$\mathrm{d}=92 \&$ Itemid $=96$

Universidad Nacional (s. f.). Manual de Perfiles de Cargos. Recuperado de www.una.ac.cr

Universidad Nacional. (2011) Modelo pedagógico universitario. Heredia: Departamento de Publicaciones de la Universidad Nacional.

Universidad Nacional. (2004). Plan global institucional 2004-2011. Heredia: Departamento de Publicaciones de la Universidad Nacional.

Universidad Nacional. (2006). Reglamento general sobre los procesos de enseñanza y de aprendizaje de la Universidad Nacional. Heredia: Departamento de Publicaciones de la Universidad Nacional.

Universidad Nacional (2012). Diccionario de competencias. Recuperado de www.una.ac.cr. 